\title{
AlN-on-Si Square Diaphragm Piezoelectric Micromachined Ultrasonic Transducer with Extended Range of Detection ${ }^{+}$
}

\author{
Suresh Alasatri 1,*, Libor Rufer ${ }^{2}$ and Joshua Lee ${ }^{1,3}$ \\ 1 Department of Electronic Engineering, City University of Hong Kong, Hong Kong, China; \\ joshulee@cityu.edu.hk \\ 2 CNRS, Grenoble INP, TIMA, University Grenoble Alpes, 38000 Grenoble, France; libor.rufer@imag.fr \\ 3 State Key Laboratory of Millimeter Waves, City University of Hong Kong, Hong Kong, China \\ * Correspondence: salasatri2-c@my.cityu.edu.hk; Tel.: +852-5134-4849 \\ † Presented at the Eurosensors 2018 Conference, Graz, Austria, 9-12 September 2018.
}

Published: 27 November 2018

\begin{abstract}
We present aluminum nitride (AlN) on silicon (Si) CMOS-compatible piezoelectric micromachined ultrasonic transducers (pMUTs) with an extended detection range of up to $140 \mathrm{~cm}$ for touchless sensing applications. The reported performance surpasses the current state-of-art for AlN-based pMUTs in terms of the maximum range of detection using just a pair of pMUTs (as opposed to an array of pMUTs). The extended range of detection has been realized by using a larger diaphragm allowed by fabricating a thicker diaphragm than most other pMUTs reported to date. Using a pair of pMUTs, we experimentally demonstrate the capability of range-finding by correlating the time-of-flight (TOF) between the transmit (TX) and receive (RX) pulse. The results were obtained using an experimental setup where the MEMS chip was interconnected with a customized printed circuit board (PCB) using $\mathrm{Al}$ wire bonds.
\end{abstract}

Keywords: aluminum nitride (AIN); piezoelectric micromachined ultrasonic transducer (pMUT); touchless sensing

\section{Introduction}

Piezoelectric micromachined ultrasonic transducers (pMUTs) have recently emerged as highly promising candidates for a range of practical applications that include fingerprint sensing, gesture recognition, range finding, non-destructive testing and medical imaging, due to their low power consumption and small form factor [1]. Compared to capacitive MUTs (cMUTs), the dynamic range of linear displacement of pMUTs is larger. This is because the capacitance of the transduction gap in cMUTs remains linear when the displacement is much smaller than the nominal gap. In the case of pMUTs, the displacement range of the diaphragm is not limited by a back plate as cavity is typically etched on the back plane of the diaphragm [2]. Piezoelectric materials that have been commonly used in pMUTs include lead zirconate titanate (PZT), zinc oxide ( $\mathrm{ZnO}$ ) and AlN [1].

AlN-based pMUTs have been of increasing interest for range-finding applications compared to PZT and ZnO films owing to their CMOS-compatibility and maturity of deposition techniques [3]. AlN-based pMUTs have been fabricated based on an AlN-on-silicon oxide bilayer [4], which results in a more compliant diaphragm compared to structures realized from stiffer substrates like silicon. These structures are limited to a detection range below $1 \mathrm{~m}$ (operating at $400 \mathrm{kHz}$ ). In this paper, with the aim of extending the maximum detectable range beyond the current state-of-art of $1 \mathrm{~m}$, we have fabricated pMUTs based on an AlN-on-silicon (Si) bilayer that stiffens the diaphragm. As a proof of concept, we have designed a pair of square diaphragm pMUTs with side lengths of $2300 \mu \mathrm{m}$ by 2300 $\mu \mathrm{m}$ fabricated on the same chip. 


\section{Device Implementation and Simulation}

We performed finite element analysis (FEA) in COMSOL for our proposed device and evaluated the resonant frequency and total strain profile of the device using an eigenfrequency study as shown in Figure 1. The proposed pMUT devices were fabricated using a standard AlN-on-Si fabrication process [5]. By fabricating the AlN transducer on a Si diaphragm, much thicker membranes can be realized compared to a membrane comprising AlN and silicon oxide (10 $\mu \mathrm{m}$ compared to $1 \mu \mathrm{m})$. The proposed pMUT diaphragm in this work comprises a thin $0.5 \mu \mathrm{m}$ AlN film on a $10 \mu \mathrm{m}$-thick Si device layer as shown in Figure 2a. A thicker diaphragm allows for large lateral dimensions for a given resonant frequency, which ultimately increases the sensitivity of the pMUT. As shown in Figure $2 b$, our pMUT comprises a square diaphragm with $1 \mu \mathrm{m}$ thick $\mathrm{Al}$ top electrodes along its clamped sides. The electrodes were used to either actuate the diaphragm (for the transmit mode) or to convert the incident pressure wave into an output current (for the receivemode).

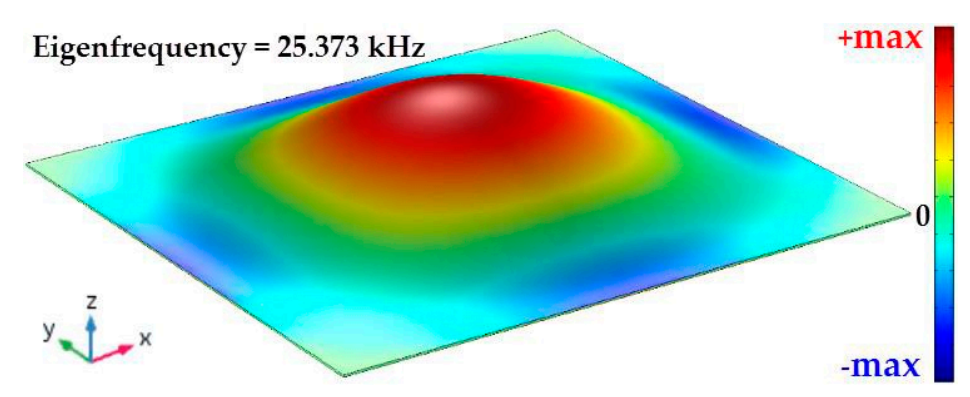

Figure 1. Deformed shape of square diaphragm and profile of the sum of lateral strain components $\left(\varepsilon_{\mathrm{x}}+\varepsilon_{\mathrm{y}}\right)$ simulated using COMSOL.

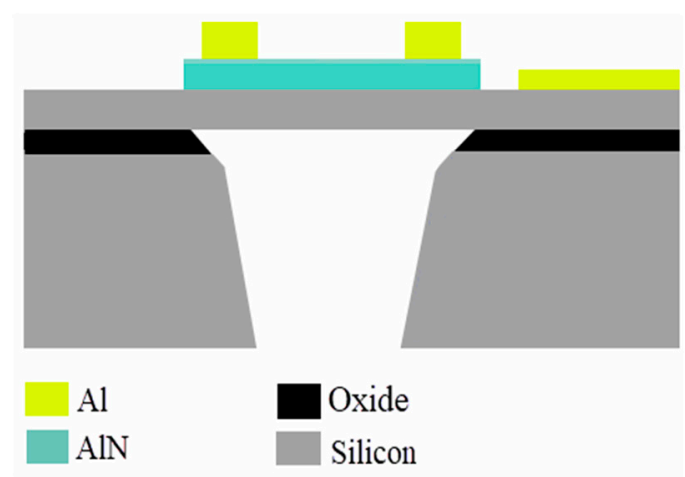

(a)

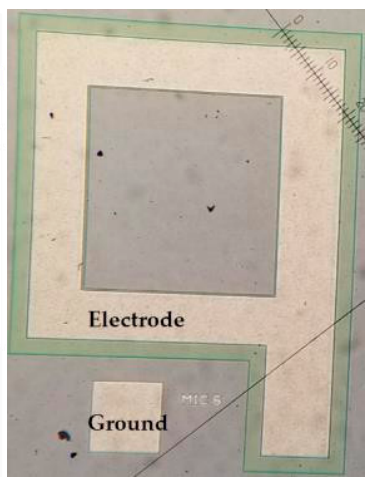

(b)

Figure 2. (a) Schematic showing a cross-sectional view of the proposed pMUT with a back cavity; (b) Optical micrograph of fabricated pMUT $(2300 \mu \mathrm{m}$ by $2300 \mu \mathrm{m})$ seen from the top side of the device.

\section{Device Characterization and Experimental Results}

\subsection{Electrical and Acoustic Characterization}

The fabricated device was mounted on a custom-designed PCB using wire bonds to connect the contact pads of the MEMS device to the PCB tracks. We measured the frequency response of the acoustic pressure from the pMUTs by placing a $1 / 4$ " free-field microphone (Brüel \& Kjær 4954) $10 \mathrm{~mm}$ from the front surface of the diaphragm. Using a Zurich Instruments HFLI lock-in amplifier, the device was swept around its resonance with an excitation voltage of $0.5 \mathrm{~V}$ applied while the output from the microphone was measured. The result is depicted in Figure 3, which shows that the resonance is around $33 \mathrm{kHz}$ with a peak sound pressure output of $2 \mathrm{~Pa}$. For comparison, [6] reported a peak sound pressure of less than 0.2 Pa despite having applied a higher excitation voltage of $3 \mathrm{~V}$. 


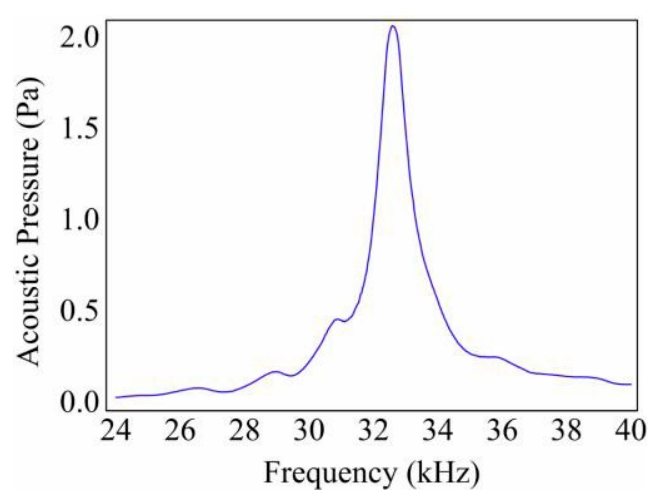

Figure 3. Frequency response of the acoustic pressure measured with a free-field microphone placed $10 \mathrm{~mm}$ from the surface of the pMUT.

\subsection{Range-Detection Demonstration and Calibration}

To demonstrate the capability of range-finding, one of the pMUTs on the chip was used to transmit (TX) an acoustic pulse while the other was used to receive (RX) the returning echo that has been reflected from a nearby obstacle placed at a calibrated distance from the pMUT. The rear side of the pMUT was used as the active side in the range-detection experiments. The RX pMUT was fed to a transimpedance amplifier to gain up the signal.

The input TX pulse comprised a sinewave at a carrier frequency of $29 \mathrm{kHz}$ in periodic bursts with an obstacle placed away from the back cavity of the pMUTs. By measuring the time-of-flight (TOF) between the TX and RX echo pulse as shown in Figure 4, the distance of an obstacle from the pMUT chip may thus be inferred. The TOF was measured as the difference between the start of the TX pulse (not shown here) and the beginning of the RX echo pulse. The beginning of the RX pulse was identified at the point when the $V_{p p}$ of the $R X$ signal just begins to be visibly larger than the $V_{p p}$ of the noise. The TOF was measured with the obstacle placed at increasing distances (maximum $140 \mathrm{~cm}$ ) from the pMUT chip to produce the linear calibration plot shown in Figure 5a. We have verified that the slope of the best fit line matches the speed of sound, demonstrating a match between theory and experiment. Figure $5 \mathrm{~b}$ depicts the extracted signal-to-noise ratio (SNR) as a function of distance. The SNR was derived by dividing the measured maximum $V_{\mathrm{pp}}$ of each respective $R X$ signal over the $V_{\mathrm{pp}}$ of the noise. For distances below $70 \mathrm{~cm}$, the number of cycles in the burst input was reduced from 40 to 10 cycles to extend the lower bound of the range of detection. By reducing the number of cycles in a given burst, the burst period is shortened at the expense of a lower output sound pressure level as the burst ends before the oscillations of the pMUT reach steady state. As such, the SNR is reduced as a tradeoff for extending the lower limit of detection down to $40 \mathrm{~cm}$.

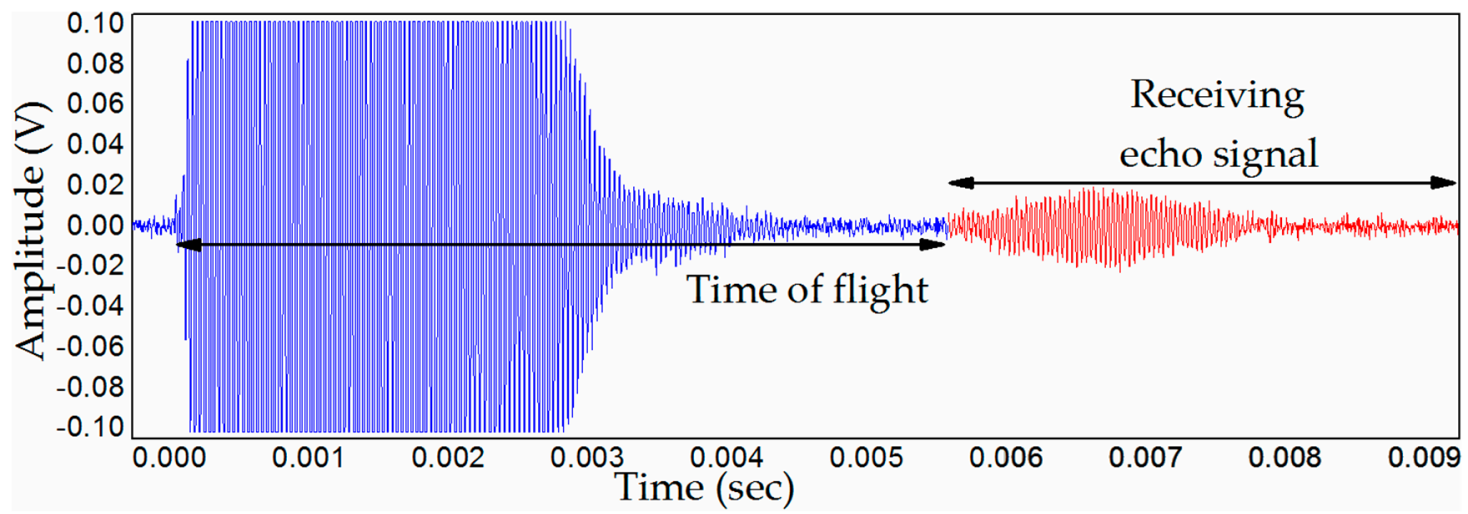

Figure 4. Measurement of time of flight (from the receiving pMUT) where the initial input burst signal (40 cycles) due to cross talk between devices is followed by a weaker receiving echo signal. 


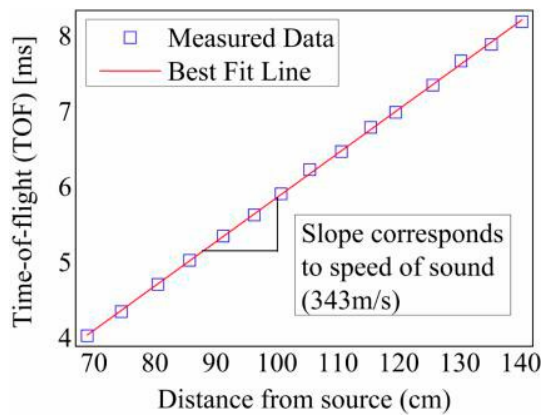

(a)

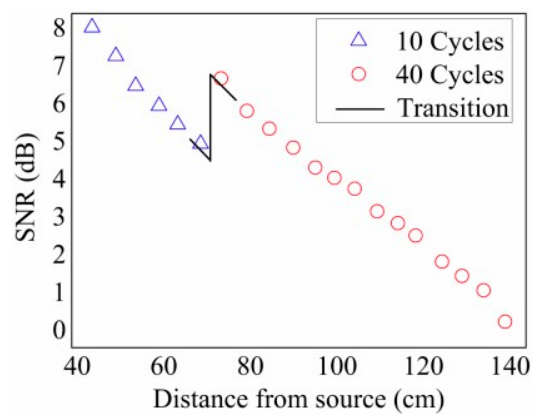

(b)

Figure 5. (a) Linear calibration measurement of round-trip time-of-flight (TOF) as a function of distance from the adjacent source on the same chip; (b) SNR of received echo vs. distance where the number of cycles in the input burst signal applied to the transmit pMUT was reduced from 40 to 10.

\section{Conclusions}

In this paper, we have designed and fabricated aluminum nitride on silicon square diaphragm piezoelectric micromachined ultrasonic transducer for touchless sensing applications. We have successfully demonstrated a wide detection range from $40-140 \mathrm{~cm}$ using a pair of square diaphragm AlN-pMUTs. The proposed device shows its potential to touchless sensing applications.

Author Contributions: A.S., L.R., and J.L. performed the experiments, L.R. designed the devices, A.S. wrote the paper, L.R. and J.L. corrected the paper.

Acknowledgments: The proposed work was supported by a grant from the Research Grants Council of Hong Kong under the project number CityU 11211116.

Conflicts of interest: The authors declare no conflicts of interest.

\section{References}

1. Przybyla, R.J.; Shelton, S.E.; Guedes, A.; Izyumin, I.I.; Kline, M.H.; Horsley, D.A.; Boser, B.E. In-air rangefinding with an AIN piezoelectric micromachined ultrasound transducer. IEEE Sensors 2011, 11, 26902697, doi:10.1109/JSEN.2011.2157490.

2 Przybyla, R.; Flynn, A.; Jain, V.; Shelton, S.; Guedes, A.; Izyumin, I.; Horsley, D.; Boser, B.A. Micromechanical ultrasonic distance sensor with $>1 \mathrm{~m}$ range. In Proceedings of the IEEE 16th International Solid-State Sensors, Actuators and Microsystems Conference (Transducers), Beijing, China, 5-9 June2011.

3. Wang, T.; Kobayashi, T.; Yang, B.; Wang, H.; Lee, C. Highly sensitive piezoelectric micromachined ultrasonic transducer (pMUT) operated in air. In Proceedings of the IEEE 11th International Conference on Nano/Micro Engineered and Molecular Systems (NEMS), Sendai, Japan, 17-20 April 2016.

4. Rozen, O.; Block, S.T.; Mo, X.; Bland, W.; Hurst, P.; Tsai, J.M.; Daneman, M.; Amirtharajah, R.; Horsley, D.A. Monolithic MEMS-CMOS ultrasonic rangefinder based on dual-electrode PMUTs. In Proceedings of the IEEE 29th International Conference on MEMS, Shanghai, China, 24-28 Jan 2016.

5. Cowen, A.; Hames, G.; Glukh, K.; Hardy, B. PiezoMUMPs Design Handbook; MEMSCAPInc.: Durham, NC, USA, 2014.

6. Guedes, A.; Shelton, S.; Przybyla, R.; Izyumin, I.; Boser, B.; Horsley, D.A. Aluminum nitride pMUT based on a flexurally-suspended membrane. In Proceedings of the IEEE 16th International Solid-State Sensors, Actuators and Microsystems Conference (Transducers), Beijing, China, 5-9 June 2011.

(C) 2018 by the authors. Licensee MDPI, Basel, Switzerland. This article is an open access article distributed under the terms and conditions of the Creative Commons Attribution (CC BY) license (http://creativecommons.org/licenses/by/4.0/). 